\title{
Contaminación de suelos por el uso de aguas residuales
}

\author{
Soil contamination through the use of wastewater \\ Contaminação do solo pelo uso de águas residuais
}

\author{
Juan de Dios, Aguilar Sánchez \\ juasajae@gmail.com \\ https://orcid.org/0000-0001-5152-5665
}

\author{
Napoleón Cubas Irigoín \\ napochota1@gmail.com \\ https://orcid.org/0000-0001-5950-7525
}

Universidad Nacional Autónoma de Chota, Cajamarca, Perú

Artículo recibido 02 de marzo 2021 / Arbitrado y aceptado 31 de marzo 2021 / Publicado 04 de mayo 2021

\section{RESUMEN}

La investigación tiene como objetivo general demostrar el indicie de contaminación de los suelos por el uso de las aguas residuales, la investigación fue de tipo experimental que consistió en recoger 5 muestras de terrenos de cultivo con el propósito de verificar el potencial de Hidrógeno $(\mathrm{pH})$, Conductividad Eléctrica (C. E.), Fosforo (P), Potasio (K), Carbono (C), Materia Orgánica, Nitrógeno $(\mathrm{N})$, el análisis mecánico, la Capacidad de Intercambio Catiónico (CIC), cantidad de Calcio $\left(\mathrm{Ca}^{+2}\right)$, magnesio $\left(\mathrm{Mg}^{+2}\right)$, Potasio $\left(\mathrm{K}^{+}\right)$, concentración de Sodio $\left(\mathrm{Na}^{+}\right)$e Hidruro de Calcio $\left(\mathrm{Ca}^{+2}+\mathrm{H}^{+}\right)$, los resultados relevantes destacan que la mayoría de muestras presentan concentraciones superpones de componentes químicos, a los propuestos por La Organización de las Naciones Unidas para la Alimentación y la Agricultura (FAO, 2018). En base a los datos se concluye que el exceso de $\mathrm{pH}$ encontrado en los suelos lo hace alcalino, el exceso de fósforo, potasio, carbono, materia orgánica y la regularidad del nitrógeno hace que los suelos no desempeñen sus funciones normales en el cultivo de la plantas y que los valores miliequivalentes por 100/g superan los límites permitidos la normatividad vigente.

Palabras clave: Caracterización; cationes; composición; contaminación suelo

ABSTRACT

RESUMO

The general objective of the investigation is to demonstrate the index of contamination of the soils due to the use of wastewater, the investigation was of an experimental type that consisted of collecting 5 samples of farmland with the purpose of verifying the potential of Hydrogen $(\mathrm{pH})$, Electrical Conductivity (EC), Phosphorus (P), Potassium (K), Carbon (C), Organic Matter, Nitrogen (N), mechanical analysis, Cation Exchange Capacity (CEC), amount of Calcium $\left(\left[\mathrm{Ca} \rrbracket^{\wedge}(+2)\right)\right.$, Magnesium $\left(\left[\mathrm{Mg} \rrbracket^{\wedge}(+2)\right)\right.$, Potassium $(\mathrm{K} \wedge+)$, Sodium concentration $\left([\mathrm{Na}]^{\wedge}+\right)$ and Calcium Hydride $(\llbracket \mathrm{Ca}) \wedge(+2)+\mathrm{H}^{\wedge}$ $+)$, the relevant results highlight that most samples present superimposed concentrations of chemical components, to those proposed by the Food and Agriculture Organization of the United Nations (FAO, 2018). Based on the data, it is concluded that the excess $\mathrm{pH}$ found in the soils makes it alkaline, the excess of phosphorus, potassium, carbon, organic matter and the regularity of nitrogen makes the soils not perform their normal functions in the cultivation of the plants and that the milliequivalent values per 100 / g exceed the limits allowed by current regulations.

Key words: Characterization; cations; composition; contamination and soil
O objetivo geral da pesquisa é demonstrar a indicação de contaminação do solo pelo uso de águas residuais, a pesquisa foi de tipo experimental que consistiu na coleta de 5 amostras de terra cultivada com o propósito de verificar o Potencial de Hidrogênio $(\mathrm{pH})$, Condutividade Elétrica (E. C.). ), Fósforo (P), Potássio (K), Carbono (C), Matéria Orgânica, Nitrogênio (N), análise mecânica, Capacidade de Troca Catiônica (CEC), quantidade de Cálcio $\left(\mathrm{Ca}^{+2}\right)$, Magnésio $\left(\mathrm{Mg}^{+2}\right)$, Potássio $\left(\mathrm{K}^{+}\right)$, Concentração de Sódio $\left(\mathrm{Na}^{+}\right)$e Hidreto de Cálcio $\left(\mathrm{Ca}^{+2}+\mathrm{H}^{+}\right)$, os resultados relevantes destacam que a maioria das amostras apresenta concentrações sobrepostas de componentes químicos, como proposto pela Organização das Nações Unidas para Alimentação e Agricultura (FAO, 2018). Com base nos dados, conclui-se que o excesso de $\mathrm{pH}$ encontrado nos solos o torna alcalino, o excesso de fósforo, potássio, carbono, matéria orgânica e a regularidade do nitrogênio fazem com que os solos não desempenhem suas funções normais no cultivo de plantas e que os valores miliequivalentes por 100/g excedam os limites permitidos pela regulamentação atual.

Palavras-chave: Caracterização; cátions; composição; contaminação do solo 


\section{INTRODUCCIÓN}

La contaminación de suelos en los valles de los ríos se produce como consecuencia del uso de aguas residuales en las actividades agrícolas en las diversas partes del planeta, las aguas provenientes de los desagües, industrias, relaves mineros que se vierten en las quebradas y ríos, transportan coliformes totales, fecales y enterobacterias, componentes físicos y químicos que al utilizarse el agua en el riego, los componentes se concentran en los suelos, alteran su composición, degradándolo y haciéndolo no apto para el cultivo. Ibañez, manifiesta que los suelos se ven contaminados cuando el pH está por encima de 8.5 como producto del incremento de sales sódicas y potásicas o menor a 4.5 muy ácidos por la pobreza de nutrientes, la contaminación de los suelos se da por efecto de las actividades humanas, el excesivo uso de fertilizantes, fosfatos, nitratos o sustancias radioactivas que transportas las aguas residuales ya sea proveniente de la industria, desagües o el uso de fungicidas en la actividad agrícola; en consecuencia la contaminación de los suelos se caracteriza por la pérdida de materia orgánica o la alteración de los componentes físicos y químicos a causa del uso de aguas residuales en el riego. (1).

La contaminación de los suelos en los valles de los ríos y quebradas de Latino América, se propicia por el uso de las aguas residuales provenientes de las diversas actividades humanas, la concentración física y química que arrastran se almacenan en los suelos, haciéndolo áridos y contaminados que necesariamente necesitan de tratamiento para ser utilizados en la agricultura. La Organización de las Naciones Unidas para la Alimentación y la Agricultura (2) señala que: ...el $14 \%$ de degradación de suelos en el mundo se da en Latino América y el Caribe, siendo Mesoamérica el territorio mas afectado con 26 $\%$, América del Sur $14 \%$, siendo las principales causas la salinización, la pérdida de carbono orgánico y la erosión de los suelos, originados por la explotación de recursos minerales, forestales, el agua, agroquímicos que aporta entre el $20 \%$ y $40 \%$ de suelos degradados. (p. 7).

Estos efectos son producidos por diversas actividades que desarrolla el hombre en la naturaleza, donde los principales desechos y compuestos químicos que produce, son arrojados en las periferias de los suelos, que luego son arrastradas por las aguas de las lluvias y residuales a las quebradas y ríos, que al ser utilizadas en el riego se concentran en los suelos y alteran la composición física y química, degradándolo y haciéndolo no apta para las actividades agrícolas.

La contaminación de los suelos en el Perú no es ajena al ámbito latinoamericano, es un país minero, agrícola y con gran cantidad de recursos forestales, y agua, sin embargo es la población quien lo utiliza de manera irresponsable, haciendo que los residuos sólidos, orgánicos o líquidos vayan a con contaminar las el agua de quebradas y ríos, que al ser utilizadas en el riego, los componentes físicos y químicos se concentran en los suelos propiciando la contaminación, que no solo trae consecuencias graves para la producción agropecuaria de los valles, sino que perjudica la salud de quienes lo consumen. Marjani y Sagasta, (3), resalta que en el suelo: 
Las aguas residuales para riego añaden nutrientes, sólidos disueltos, sales y metales pesados al suelo. Con el tiempo pueden acumularse cantidades excesivas de estos elementos en la zona de raíces, lo que puede ser perjudicial para el suelo. El uso de aguas residuales a largo plazo podría ocasionar en el suelo: salinidad, sobresaturación, desintegración de su estructura, una reducción generalizada de su capacidad productiva y reducir el rendimiento de los cultivos. Las consecuencias dependerán de factores como la fuente, la intensidad del uso y la composición de las aguas residuales, así como de las propiedades del suelo y las características biofísicas propias de cada cultivo. (p. 78).

Los datos demuestran que la acumulación de elementos químicos orgánicos e inorgánicos en las aguas residuales que se transportan en las quebradas y ríos por efecto de la actividad minera, industrial y actividad humana contamina los suelos de los valles cuando se utiliza en el riego, la producción constituye un peligro para la salud de los seres vivos.

Cajamarca por ser una región productora de minerales, parte de sus suelos se ven contaminados con elementos químicos provenientes de los relaves, que son arrojados a las aguas naturales de los ríos, y estas al mezclarse y ser utilizadas como riego en los diversos valles de cultivo se van acumulando en los suelos haciéndolo infértiles, por otro lado el uso de las aguas residuales que proviene de las ciudades en su recorrido recogen desagües y residuos sólidos que al descomponerse, contaminan el agua y su uso en el riego contaminan el suelo. Noticias Ser, hace constar que en Cajamarca diariamente se recogen 140 toneladas de residuos sólidos: "Los sólidos al interior de las aguas van produciendo peligrosos compuestos químicos, mientras que las aguas residuales van concentrando coliformes". Lo expuesto determina que los valles de las quebradas y ríos de la región concentran aguas servidas con residuos sólidos, cuya descomposición no solo contamina los suelos y las plantas donde se utiliza como riego, sino que pone en grave riesgo la salud de las personan que consuman la producción agrícola (4).

Los valles de las quebradas y ríos aledaños a las ciudades en la provincia, se ven contaminados por las acciones irresponsables del hombre, se tiene problemas de contaminación de producción, ya sea por el uso de fertilizantes, pesticidas, sales, sedimentos, metales, materia orgánica, patógenos y contaminantes emergentes que conducen las aguas residuales y que al utilizarse en el riego se acumulan en los suelos contaminándolo, este efecto se observa en los valles de las quebradas San Mateo, San Juan, Colpa Mayo y el mismo río Chotano, donde sus suelos se ven alterados por la presencia de componentes físicos y químicos que contaminan la producción, ante el problema la investigación trata de demostrar el índice de contaminación que presentan los suelos por efecto de las aguas residuales.

Existen diversos estudios que trata el tema de esta investigación donde se puede resaltar que los estudios realizados por Guadarrama y Galván, resalta que el crecimiento de la población y el desarrollo industrial en las ciudades sobreexplota el agua y el suelo propiciando su contaminación a traves de la proliferación de aguas residuales y residuos sólidos que lamentablemente contaminan los suelos al ser utilizados como riego en la agricultura (5). 
De la misma manera, en investigaciones realizadas por Rodríguez, McLaughlin, y Pennock, en su estudio resaltan que en el mundo unos 22 millones de hectareas de suelos están contaminados, es así que en China el $19 \%$ de los suelos estarian contaminados, en Europa existirían unos 3 millones de emplazamientos contaminados, en los Estados Unidos habrían unos 1300 emplazamientos de contaminación y en Australia unos 80000 emplazamientos de contaminación, ante los resultados se hace necesario la urgente intervención de las organizaciones internacionales para detener la contaminación de los suelos (6).

Otro antecedente de esta investigacion es la realizada por Mendoza, donde trata sobre Evaluación fisicoquímica de la calidad del agua superficial en el Centro Poblado de Sacsamarca, región Ayacucho, los fosfatos presentan 1.51 ppm, el arsénico 0.13 ppm, los cuales demuestran que hay una importante concentración de arsenio en el río Caracha, y que su utilidad en el riego contamina los suelos, los resultados significan una alerta para las autoridades porque este parámetro es un indicador de eutrofización y contaminación (7). Larios, González, y Morales manifiesta que el 70\% de aguas residuales en el país no tienen tratamiento, y constituyen un riesgo para ser utilizados en el riego artesanal, concentran altos índices de componentes físicos y químicos que contaminan los suelos, de las 143 plantas de tratamiento residual, solo el 14 \% cumplen con los parámetros establecidos en la normatividad vigente (8).

En este sentido, Soriano señala que en el análisis de las 3 muestras de aguas subterráneas se encuentran estándares no aceptables para el uso de riego de verduras, ni para el consumo de las personas y animales, al encontrarse excesiva cantidad de coliformes totales y termotolerantes, tendría que tratarse antes de ser utilizada (9). Díaz y Medina determinan que hay mayor concentración de Magnesio (Mg) en las plantas con 2997 mg/ $\mathrm{kg}$, la planta que retuvo más metales fue el cacalioides ambos grupos, el metal que más se acumula en los suelos es el hierro con 65020 $\mathrm{mg} / \mathrm{kg}$ en el punto de muestra 2, demuestra que, si el suelo concentra gran cantidad de minerales, las plantas también lo acumulan (10). Huamaní, en su estio establece que el uso continuo de aguas servidas provoca el detrioro físico y químico de los suelos, al demostrar que con el análisis microbiológico existe elevada cantidad microbiana en el suelo y en el agua, estos se concentran tambien en los cultivos, por lo tanto concluye que con los resultados se debe informar a los agricultores fomentar las buenas prácticas agrícolas a fin de obtener una produción sana y sin contaminación. Por otra parte, encuentra que las aguas al igual que los suelos presentan altas concentraciones de $\mathrm{P}, \mathrm{K}, \mathrm{CaCO} 3$, metales pesados los cuales constituyen agentes contaminantes (11).

El desarrollo del estudio de la contaminación de suelos por el uso de aguas residuales fue necesario, porque la población de la ciudad de Chota necesita conocer el índice de contaminación de los suelos de los valles de las quebradas San Mateo, Colpa Mayo, San Juan y río Chotano, debido que las tierras de los valles aledaños a la ciudad son utilizados por los pobladores para la producción agropecuaria y los excedentes colocados en el mercado local.

Las fundamentaciones de las bases teóricas de la investigación se sustentan en los estudios hechos por la Organización de las Naciones Unidas para la Alimentación (2-11) que consideran los siguientes parámetros para el estudio de la calidad de los suelos: 
Tabla 1. Estándares para calidad de los suelos.

\begin{tabular}{|c|c|c|c|c|c|}
\hline & \multirow[t]{2}{*}{ Indicadores } & \multirow[t]{2}{*}{ U. M } & \multirow[t]{2}{*}{ Clasificación } & \multicolumn{2}{|c|}{$\begin{array}{l}\text { Valores de Calidad } \\
\text { de los Suelos }\end{array}$} \\
\hline & & & & Valor mínimo & Valor máximo \\
\hline \multirow{11}{*}{ Parámetros } & \multirow{7}{*}{$\begin{array}{l}\text { pH (potencial } \\
\text { de Hidrógeno }\end{array}$} & \multirow{7}{*}{ Unidad } & Fuertemente ácido & $<5,5$ & \\
\hline & & & Moderadamente ácido & 5,6 & 6,0 \\
\hline & & & Ligeramente ácido & 6.1 & 6.5 \\
\hline & & & Neutro & 6.6 & 7.0 \\
\hline & & & Ligeramente alcalino & 7.1 & 7.8 \\
\hline & & & Moderadamente alcalino & 7.9 & 8.4 \\
\hline & & & Fuertemente alcalino & $>8,5$ & \\
\hline & \multirow{4}{*}{$\begin{array}{c}\text { C.E. } \\
\text { (Conductividad } \\
\text { Eléctrica) }\end{array}$} & \multirow{4}{*}{$\begin{array}{l}\text { mS/m (milésima } \\
\text { por metro) }\end{array}$} & No salino & $<2$ & \\
\hline & & & Ligeramente salino & 2 & 4 \\
\hline & & & Salino & 4 & 8 \\
\hline & & & & $>8$ & \\
\hline \multirow{17}{*}{ Parámetros } & \multirow{3}{*}{ P (Fosforo) } & \multirow{3}{*}{$\begin{array}{c}\text { ppm } \\
\text { (partes por millón) }\end{array}$} & Bajo & $<7.0$ & \\
\hline & & & Medio & 7.0 & 14 \\
\hline & & & & $>14$ & \\
\hline & & & Bajo & $<100$ & \\
\hline & K (Potasio) & ppm & Medio & 100 & 240 \\
\hline & & & & $>240$ & \\
\hline & & & Bajo & $<0.9$ & \\
\hline & C (carbono) & $\%$ & Normal & 0,9 & 1,15 \\
\hline & \multirow{4}{*}{$\begin{array}{l}\text { M.O (Materia } \\
\text { orgánica) }\end{array}$} & & & $>1,5$ & \\
\hline & & \multirow{3}{*}{$\%$} & Bajo & $<2.0$ & \\
\hline & & & Medio & 2 & 4 \\
\hline & & & Alto & $>4.0$ & \\
\hline & \multirow{5}{*}{ N (Nitrógeno) } & \multirow{5}{*}{$\%$} & Muy pobre & 0.00 & 0.10 \\
\hline & & & Pobre & 0.10 & 0.15 \\
\hline & & & Medianamente ricos & 0,15 & 0.25 \\
\hline & & & Ricos & 0.25 & 0.30 \\
\hline & & & Muy ricos & $>0.30$ & \\
\hline
\end{tabular}

Fuente: (11-12).

Para el análisis mecánico de los suelos y la capacidad de intercambio catiónico como base teórica se utilizó los indicadores establecidos por la FAO y los establecidos por Andrades y
Martínez, las cuales están en concordancia con las normas nacionales (2-12), tal como se detallan a continuación: 
Tabla 2. Estándares para calidad de los suelos.

\begin{tabular}{|c|c|c|c|c|c|}
\hline & \multirow[t]{2}{*}{ Indicadores } & \multirow[t]{2}{*}{ U. M } & \multirow[t]{2}{*}{ Clasificación } & \multicolumn{2}{|c|}{$\begin{array}{l}\text { Valores de Calidad } \\
\text { de los Suelos }\end{array}$} \\
\hline & & & & Valor mínimo & Valor máximo \\
\hline & & & Muy bajo & $<0.7$ & \\
\hline & & & Bajo & 0.7 & 1.2 \\
\hline & Arena & $\%$ & Normal & 1.2 & 1.7 \\
\hline & & & Alto & 1.7 & 2.2 \\
\hline & & & Muy Alto & $>2.2$ & \\
\hline & & & Muy bajo & $<1.0$ & \\
\hline & & & Bajo & 1.0 & 1.5 \\
\hline \multirow[t]{9}{*}{$\begin{array}{l}\text { Análisis } \\
\text { mecánico }\end{array}$} & Limo & $\%$ & Normal & 1.5 & 2.0 \\
\hline & & & Alto & 2.0 & 2.5 \\
\hline & & & Muy Alto & $>2.5$ & \\
\hline & & & Muy bajo & $<1.2$ & \\
\hline & & & Bajo & 1.2 & 1.7 \\
\hline & Arcilla & $\%$ & Normal & 1.7 & 2.2 \\
\hline & & & Alto & 2.2 & 3.0 \\
\hline & & & Muy Alto & $>3.0$ & \\
\hline & & & Suelos arenosos & 5 & \\
\hline \multirow{2}{*}{\multicolumn{2}{|c|}{$\begin{array}{c}\text { CIC (Capacidad de } \\
\text { intercambio Catiónico) }\end{array}$}} & meq/100g & Suelos francos & 5 & 15 \\
\hline & & & Suelos arcillosos & 15 & 25 \\
\hline
\end{tabular}

Fuente: (11-12).

Los suelos estudiados por ser aprovechados para la producción agropecuaria utilizando las aguas residuales se presumieron que están contaminados, para el análisis de cationes cambiables de los suelos se tomaron las muestras correspondientes y los resultados se compararon con los indicadores establecidos por la FAO y Andrades y Martínez (2-12), los párametros se presentan en el cuadro de acontinuación: si esta 
Tabla 3. Cationes cambiables de los suelos.

\begin{tabular}{cccccc}
\hline & Indicadores & U.M & Clasificación & \multicolumn{2}{c}{$\begin{array}{c}\text { Valores de Calidad } \\
\text { de los Suelos } \\
\text { Valor mínimo Valor máximo }\end{array}$} \\
\hline \multirow{2}{*}{$\begin{array}{c}\text { Cationes } \\
\text { cambiables }\end{array}$} & $\mathrm{Ca}^{+2}$ (Calcio) & Meq/100g & Normal & 60 & 75 \\
& $\mathrm{Kg}^{+2}$ (magnecio) & Meq/100g & Normal & 15 & 20 \\
& $\mathrm{Na}^{+}$(Sodio) & Meq/100g & Normal & 3 & 7 \\
& $\mathrm{Al}^{* 3}+^{*}$ (acidez) & Meq/100g & Normal & $>15$ & $<5.5$ \\
\hline
\end{tabular}

Fuente: (11-12).

En este sentido, esta investigación tiene como objetivo general demostrar el índice de contaminación de suelos por el uso de aguas residuales.

\section{METODOLOGÍA}

La investigación se desarrolló en los valles de las quebradas, San Mateo, Colpa Mayo, San Juan y Río Chotano, ubicados en los alrededores de la ciudad de Chota, departamento de Cajamarca, el área de estudio partió del primer punto de muestra a 2388 m.s.n.m. de altitud en la quebrada Colpa Mayo, hasta 2302 m.s.n.m., en la unión con el Río Chotano, siguiendo el curso de 3 Km., hasta los 2257 m.s.n.m. en la desembocadura de la quebrada San Mateo, siguiendo el recorrido aguas arriba hasta los 2412 m.s.n.m., en el punto de encuentro con las aguas de la quebrada San Juan a 2360 m.s.n.m. recorriendo hasta los 2289 m.s.n.m.; la distancia total es de $10.03 \mathrm{Km}$.

Entre los materiales utilizados se destacan: 01 estación total, 02 prismas, 01 GPS satelital, 01 wincha de 5 metros, una brújula, 01 corrector, movilidad, esmalte y brochas para ir marcando los puntos de muestra y el perímetro de los terrenos de cultivo para el análisis de los suelos, para la recolección de muestras se utilizaron bolsas especiales llevadas al laboratorio para su respectivo análisis. El levantamiento topográfico se hizo ubicando los puntos de muestreo de las aguas residuales, estableciendo un radio de 50 por 30 metros de los terrenos de cultivo utilizados para la agricultura.

La investigación fue de diseño experimental, con un nivel descriptivo cualitativo, basándose en los objetivos específicos: diagnosticar la caracterización de los suelos a causa del uso de las aguas residuales y determinar la acumulación de agentes contaminantes en los suelos de los valles de las quebradas San Mateo, Colpamayo, San Juan y Río Chotano por el uso de las aguas residuales provenientes de la zona urbana de Chota, para el análisis de calidad de los suelos, la composición mecánica de los suelos y cationes cambiables de los suelos se tuvo en cuenta los indicadores de calidad establecidos para el país y de Organización de las Naciones Unidas para la Alimentación FAO y los estudios aplicados por Andrades y Martínez, (2-12).

La población lo constituyeron los suelos que utilizan los colectores de aguas residuales que desembocan en las quebradas San Mateo, Colpa Mayo, San Juan y río Chotano en un radio de 50 por 30 metros y que en tiempo de verano son 
utilizadas para irrigar las producción agrícola en cada punto crítico, los suelos y sedimentos fueron seleccionados a fin de conocer la calidad toxológica.

La muestra fue seleccionada intencionalmente y estuvo representada por 5 puntos críticos que utilizan las aguas residuales para irrigar la producción agrícola en una malla de 50 por 30 metros.

Como técnica de recolección de datos se utilizó la observación, permitió identificar los puntos críticos para la recolección de muestras y el análisis en el laboratorio y el registro correspondiente de los resultados que se presentan en los cuadros respectivos.
Como instrumento se utilizó la ficha de registro, que permitió describir los resultados y analizarlos en comparación con las normas emitidas (2-12).

\section{RESULTADOS Y DISCUSIÓN}

Posterior a la recolección y análisis de los resultados se procedió a establecer por medio de Tablas la información obtenida en los análisis realizados, en la Tabla 4 se muestra la caracterización de los suelos de las cuencas de las Quebradas San Mateo, Colpamayo, San Juan y Rio Chotano.

Tabla 4. Caracterización de los suelos de las cuencas de las quebradas San Mateo, Colpamayo, San Juan y Río Chotano.

\begin{tabular}{ccccccccccc}
\hline $\begin{array}{c}\mathbf{N}^{\circ} \text { de } \\
\text { muestra }\end{array}$ & $\begin{array}{c}\mathbf{p H} \\
(\mathbf{1 : 1 )}\end{array}$ & $\begin{array}{c}\mathbf{C . E}(\mathbf{1 . 1}) \\
\mathbf{m S} / \mathbf{m}\end{array}$ & $\mathbf{P}$ & $\mathbf{K}$ & $\mathbf{C}$ & $\mathbf{M . 0}$ & $\mathbf{N}$ & Arena & Limo & Arcilla \\
& 8.26 & 0.54 & 13.60 & 437.99 & 2.42 & 4.18 & 0.21 & 38.0 & 20.0 & 42.0 \\
\hline 1 & 8.18 & 0.37 & 30.15 & 489.05 & 2.67 & 4.60 & 0.23 & 74.0 & 12.0 & 14.0 \\
2 & 8.30 & 0.40 & 25.43 & 509.78 & 2.18 & 3.76 & 0.19 & 26.0 & 24.0 & 50.0 \\
3 & 8.01 & 0.24 & 37.75 & 485.24 & 3.98 & 6.85 & 0.34 & 70.0 & 10.0 & 20.0 \\
4 & 7.44 & 0.39 & 34.86 & 501.59 & 4.02 & 6.94 & 0.35 & 76.0 & 8.0 & 16.0 \\
\hline 5 & & & & & & & & & & \\
\hline
\end{tabular}

El análisis de las muestras de los suelos en un radio comprendido entre $50 \mathrm{~m}$ y $30 \mathrm{~m}$ de los puntos más críticos, indican que la medida del potencial de Hidrógeno $(\mathrm{pH})$ de la suspensión del suelo: agua relación 1.1 de las 5 muestras se distribuye entre 7.44 y 8.30 los valores demuestran que el suelo de la muestra 5 tiene $\mathrm{pH}$ de 7.44 ligeramente neutro o suelo básico, mientras que el resto de muestras tienen $\mathrm{pH}$ por encima de lo establecido por la norma nacional.

La Conductividad Eléctrica (C. E.) del extracto acuoso concentrada en las muestras señaladas se tiene parámetros comprendidos de $0.24 \mathrm{dS} / \mathrm{m}$ a $0.54 \mathrm{dSm}$ el cual determina que los suelos son ligeramente muy salinos.

El Fosforo (P) acumulado en los suelos según muestras establecidas se distribuye de 13.60 ppm a 37.75 ppm; los resultados indican que los parámetros obtenidos en todas las muestras está por encima de la clasificación normal de los terrenos arenosos.

El Potasio (K) concentrado en cada una de las muestras está en el rango de 437.99 ppm a 509.78 ppm, los resultados de las muestras indican que se supera los límites permitidos por las normas establecidas. 
El Carbono (C) acumulado en cada una de las muestras se distribuyen de $2.18 \%$ a $6.94 \%$, valores que determinan que los suelos tienen una clasificación baja, se trata de suelos ácidos y que deberían ser tratados para darle uso agrícola.

La Materia Orgánica concentrada en las muestras se distribuye de $3.76 \%$ a $6.94 \%$, indica que supera los límites establecidos por las normas nacionales e internacionales.

El Nitrógeno $(\mathrm{N})$ existente en cada muestra está comprendido de $0.19 \%$ a $0.35 \%$, valores que indican que el nivel de disponibilidad va entre mediano, rico y muy rico.

Los resultados del análisis mecánico de los suelos indican que el valor de la muestra 1 es de $38.0 \%$ de arena, $20.0 \%$ de limo y 42.0
$\%$ de arcilla y se trata de un suelo arcilloso; los parámetros de la muestra 2 presenta $26.0 \%$ de arena, $24.0 \%$ de limo y $50 \%$ de arcilla; el valor de la muestra 3 indica que se trata de un suelo franco arenoso al obtener parámetros de $74 \%$ de arena, $12 \%$ de limo y $14 \%$ de arcilla; los suelos de la muestra 4 con valores de $70.0 \%$ de arena, $\mathbf{1 0 . 0 \%}$ de limo y $20.0 \%$ de arcilla, así como el terreno de la muestra 5 que presenta valores de $76 \%$ de arena, $8 \%$ de limo y $16.0 \%$ de arcilla demuestran que son terrenos franco arenosos tal como lo fundamenta la FAO (2).

De la misma manera en la Tabla 5 se muestra la capacidad de intercambio catiónico de los suelos de los valles de las quebradas San Mateo, Colpamayo, San Juan y Río Chotano.

Tabla 5. Capacidad de Intercambio catiónico de los suelos de los valles de las quebradas San Mateo, Colpamayo, San Juan y Río Chotano.

\begin{tabular}{|c|c|c|c|c|c|c|c|c|c|c|}
\hline \multirow{2}{*}{$\begin{array}{c}\mathbf{N}^{\circ} \text { de } \\
\text { muestra }\end{array}$} & \multirow{2}{*}{$\begin{array}{c}\text { Clase } \\
\text { textural }\end{array}$} & \multirow[t]{2}{*}{$\mathrm{ClC}$} & \multicolumn{5}{|c|}{ Cationes Cambiantes } & \multirow{2}{*}{$\begin{array}{l}\text { Suma de } \\
\text { Cationes }\end{array}$} & \multirow{2}{*}{$\begin{array}{c}\text { Suma de } \\
\text { bases }\end{array}$} & \multirow{2}{*}{$\begin{array}{l}\text { \% Sat. } \\
\text { de Bases }\end{array}$} \\
\hline & & & $\mathrm{Ca}+2$ & $\begin{array}{c}M g+ \\
M e q / 100 g\end{array}$ & $\mathbf{K}+$ & $\mathrm{Na}+$ & $\mathrm{Ca}+2+\mathrm{H}$ & & & \\
\hline 1 & Ar. & 32.11 & 28.06 & 2.83 & 1.04 & 0.18 & 0.00 & 32.11 & 32.11 & 100 \\
\hline 2 & Ar. & 26.56 & 22.64 & 2.78 & 0.88 & 0.26 & 0.00 & 26.56 & 26.56 & 100 \\
\hline 3 & Ar. & 29.81 & 25.40 & 2.99 & 1.24 & 0.18 & 0.00 & 29.81 & 29.81 & 100 \\
\hline 4 & Fr. A. & 28.60 & 25.09 & 2.32 & 1.06 & 0.14 & 0.00 & 28.60 & 28.60 & 100 \\
\hline 5 & Fr. A. & 30.32 & 25.17 & 3.95 & 1.06 & 0.14 & 0.00 & 30.32 & 30.32 & 100 \\
\hline
\end{tabular}

La Capacidad de Intercambio Catiónico (CIC) de los suelos indican que se obtienen valores miliequivalentes por $100 \mathrm{~g}(\mathrm{meq} / 100 \mathrm{~g})$ según muestras establecidas de 26.56 meq/100g a 32.11 meq/100g demostrando que supera a los límites permitidos por la normatividad internacional, FAO, (2).

En cuanto a la cantidad de Calcio ( $\left.\llbracket \mathrm{Ca} \rrbracket^{\wedge}(+2)\right)$ concentrado en cada muestra y que se distribuye entre $22.64 \%$ - $28.08 \%$, los valores determinan que tiene alta disponibilidad de ( $\left.\llbracket \mathrm{Ca} \rrbracket^{\wedge}(+2)\right)$ al superar el límite permitido por la FAO, (2).

El magnesio $\left(\left[\mathrm{Mg} \rrbracket^{\wedge}(+2)\right)\right.$ encontrado en la muestras de los suelos alcanzan un valor distribuido entre $2.32 \%$ - $3.95 \%$ resultados que indican que hay una alta concentración de $\left[\mathrm{Mg} \rrbracket^{\wedge}(+2)\right.$ demostrando que hay una alta 
disposición para destinarlo al cultivo los suelos.

La acumulación de Potasio $\left(\mathrm{K}^{\wedge}+\right)$ en los suelos de las riberas de las quebradas y ríos en estudio según muestras establecidas indican que se tiene valores entre $0.88 \%-1.06 \%$, indica que los suelos tienen una disponibilidad muy alta, tomando los parámetros establecidos por la $\mathrm{FAO},(2)$.

La concentración de Sodio $\left([\mathrm{Na}]^{\wedge}+\right)$ en los suelos de las muestras tienen un valor comprendido entre $0.14 \%$ y $0.26 \%$, los datos demuestran que hay una baja concentración de $\left[\mathrm{Na} \rrbracket^{\wedge}+\right.$ por ser $<1.5 \%$ establecido por la FAO (2).

El Hidruro de Calcio $\left(\llbracket \mathrm{Ca} \rrbracket^{\wedge}(+2)+\mathrm{H}^{\wedge}+\right)$ en las muestras de los suelo no albergan a este elemento químico, por lo que no hay riesgo que los terrenos sean alcalinos.

La suma de cationes de cada muestra, se tiene valores de $26.56 \mathrm{meq} / 100 \mathrm{~g}$ - $32.11 \mathrm{meq} / 100 \mathrm{~g}$ el cual demuestra que los suelos tienen alta disponibilidad para el cultivo de plantas.

Las muestras de los suelos resaltan que la medida del potencial de Hidrógeno $(\mathrm{pH})$ de la suspensión del suelo de las 5 muestras se distribuye entre 7.44 y 8.30 , los valores demuestran que el suelo de la muestra 5 tiene $\mathrm{pH}$ de 7.44 ligeramente neutro o suelo básico, el resto presenta $\mathrm{pH}$ por encima de lo establecido por la norma nacional, se establece que el $\mathrm{pH}$ comprendido entre 6.6 y 7.3 es neutro como mejor valor para los terrenos de cultivo, mientras que los valores menores 6.5 indica que el suelo va des ligeramente ácido hasta ultra ácido y dificulta el desarrollo de la mayoría de cultivos ya que permite la retención de los nutrientes y los límites superiores a 7.4 dificulta el desarrollo de los cultivos por tratarse de suelos alcalinos.

La Conductividad Eléctrica (C. E.) del extracto acuoso concentrada en las muestras señaladas presenta parámetros comprendidos entre 0.24
$\mathrm{dS} / \mathrm{m}$ y $0.54 \mathrm{dSm}$, determina que los suelos son muy ligeramente salinos. Ruiz (2016) señala que cuando la C.E de los suelos es $<2 \mathrm{dS} / \mathrm{m}$ no son salinos, cuando la C.E va de $2 \mathrm{dSm}-4 \mathrm{dSm}$ son suelos muy ligeramente salinos, de $4 \mathrm{dSm}$ - $8 \mathrm{dSm}$ son ligeramente salinos, de $8 \mathrm{dSm}-16$ $\mathrm{dSm}$ los suelos son moderadamente salinos y $>16$ un terreno es fuertemente salino, siendo inapropiados para el cultivo de las plantas.

El Fosforo $(P)$ que acumulan los suelos de las muestra oscilan entre 13.60 ppm y 37.75 ppm; los resultados demuestran que están por encima de la clasificación normal de los terrenos arenosos. Andrades y Martínez, establecen que el límite para este tipo de suelos es de 9 ppm - 12 ppm, en cuanto a los terrenos francos solo la muestra 2 está en el límite normal con 13.60 ppm puesto que el límite permitido es de 13 ppm a 18 ppm, mientras teniendo en cuenta los parámetros normales de fosforo para terrenos arcillosos los valores de las otras 4 muestras superan el límite permitido de 16 ppm a 24 ppm, puesto que los valores se distribuyen entre 25.43 ppm - 37.75 ppm. (12).

El Potasio (K) que concentra cada muestra está en el rango de 437.99 ppm y 509.78 ppm, los cuales indican que se supera el límite permitido por las normas establecidas. Andrades y Martínez, establecen que los valores normales permitidos para terrenos arenosos son de 96 ppm a 135 ppm, para terrenos francos de 126 ppm a 195 ppm y para terrenos arcillosos de 156 ppm a 255 ppm, los resultados indican que los suelos no desempeñan sus distintas funciones en el cultivo de las plantas. (12).

El Carbono (C) acumulado en cada una de las muestras fluctúa entre $2.18 \%$ y $6.94 \%$, los valores determinan que los suelos tienen una clasificación baja, se trata de suelos ácidos y que deberían ser tratados para darle uso agrícola, los parámetros para terrenos normales según 
Andrades y Martínez es de $10 \%$ - $20 \%$, mientras que los suelos que tienen carbono $<5 \%$ son muy bajos, de $5 \%-10 \%$ son bajos, de $10 \%-20 \%$ normales, de $20 \%$ - $40 \%$ alto y $>40 \%$ muy alto. (12).

La Materia Orgánica concentrada en las muestras se distribuye entre $3.76 \%$ y $6.94 \%$, indica que supera los límites establecidos por las normas nacionales e internacionales. Andrades y Martínez indican que en terrenos arenosos la cantidad de materia orgánica debe estar comprendida entre $1.2 \%-1.7 \%$; en terrenos francos el límite es de $1.5 \%$ y $2.0 \%$ y en terrenos arcillosos de $1.7 \%-2.2 \%$. (12)

El Nitrógeno $(\mathrm{N})$ que acumula cada muestra se distribuye entre $0.19 \%$ y $0.35 \%$, los valores indican que el nivel de disponibilidad va entre mediano, rico y muy rico. La Organización de las Naciones Unidas para la Alimentación y la Agricultura establece que los suelos que tienen parámetros de nitrógeno entre $0.15 \%-0.25 \%$ son medianamente ricos, los que concentran nitrógeno entre $0.25 \%-0.30 \%$ son ricos y los que albergan nitrógeno mayor a $0.30 \%$ son muy ricos. (2).

El análisis mecánico establece que el valor de la muestra 1 es de $38.0 \%$ de arena, $20.0 \%$ de limo y $42.0 \%$ de arcilla, se trata de un suelo arcilloso; los parámetros de la muestra 3 que presenta $26.0 \%$ de arena, $24.0 \%$ de limo y 50 $\%$ de arcilla; el valor de la muestra 2 indica que se trata de un suelo franco arenoso al obtener parámetros de $74 \%$ de arena, $12 \%$ de limo y $14 \%$ de arcilla, los suelos de la muestra 4 con valores de $70.0 \%$ de arena, $10.0 \%$ de limo y 20.0 $\%$ de arcilla; así como el terreno de la muestra 5 presenta valores de $76 \%$ de arena, $8 \%$ de limo y $16.0 \%$ de arcilla demuestran que son terrenos franco arenosos, tomando como fundamento La FAO, (2).
La Capacidad de Intercambio Catiónico (CIC) indica que los valores miliequivalentes por 100 $\mathrm{g}$ (meq/100g) obtenidos en las muestras es de $26.56 \mathrm{meq} / 100 \mathrm{~g}$ a $32.11 \mathrm{meq} / 100 \mathrm{~g}$, demuestra que supera a los límites permitidos. La FAO, (2) establece que los parámetros para suelos arenosos son de $5 \mathrm{meq} / 100 \mathrm{~g}$, para suelos francos de 5 a $15 \mathrm{meq} / 100 \mathrm{~g}$ y suelos arcillosos de 15 a 25 meq/100g. Los datos demuestran que los suelos tienen disponibilidad baja para la agricultura cuan la $\mathrm{CIC}$ es $<10$ meq/100g, disponibilidad media cuando la CIC está entre los parámetros de 10-20 meq/100gy un alto nivel de disponibilidad cuando la $\mathrm{CIC}$ es $>20 \mathrm{meq} / 100 \mathrm{~g}$.

La cantidad de Calcio $\left(\llbracket \mathrm{Ca} \rrbracket^{\wedge}(+2)\right)$ concentrado en cada muestra se distribuye entre $22.64 \%$ - $28.08 \%$, los valores determinan que hay una alta disponibilidad de $\left(\llbracket \mathrm{Ca} \rrbracket^{\wedge}(+2)\right)$, supera el límite permitido por la FAO, (2) señala que un nivel de disponibilidad alto de $\llbracket \mathrm{Ca} \rrbracket^{\wedge}(+2)$ es cuando se tiene acumulaciones $>6 \%$.

El magnesio $\left(\left[\mathrm{Mg} \rrbracket^{\wedge}(+2)\right)\right.$ encontrado en la muestras de los suelos se distribuyen entre $2.32 \%-3.95 \%$ demostrando que hay una alta concentración de $\llbracket \mathrm{Mg} \rrbracket^{\wedge}(+2)$. La FAO, (2) establece que cuando se concentra $\llbracket \mathrm{Mg} \rrbracket^{\wedge}(+2)$ $<0.4 \%$ el nivel de disponibilidad del suelo es bajo, de $0.4-0.8 \%$ es medio y cuando la concentración es $>0.8 \%$ hay un alta disposición para destinarlo los suelos al cultivo.

El Potasio $\left(K^{\wedge}+\right)$ acumulado en los suelos de las riberas de las quebradas y ríos en estudio son de $0.88 \%$ - $1.06 \%$, indica que los suelos tienen una disponibilidad muy alta para el cultivo. La FAO establece que cuando los suelos presentan parámetros $<0.12 \%$ tienen disponibilidad baja para el cultivo, la acumulación de $0.12-0.3 \%$ dan una disponibilidad media y cuando es $>0.3$ $\%$ tienen un nivel de disponibilidad alto para el desarrollo de las actividades agrícolas. (2). 
La concentración de Sodio $\left([\mathrm{Na}]^{\wedge}+\right)$ en los suelos de las muestras tienen un valor de $0.14 \%-0.26 \%$, los datos demuestran que hay una baja concentración de $\llbracket \mathrm{Na} \rrbracket^{\wedge}+$ por ser $<1.5$ $\%$. La FAO, (2) señala que cuando los valores son $<1.5 \%$ los suelos tienen disponibilidad baja para la agricultura, cuando los valores están comprendidos entre 1.5 - 3.0 \% hay una disponibilidad media y cuando los valores superan el $3.0 \%$ los suelos tienen un alto nivel de disponibilidad para utilizarlo en la agricultura.

La suma de cationes de cada muestra, presenta valores de $26.56 \mathrm{meq} / 100 \mathrm{~g}$ a 32.11 meq/100g, demuestra que los suelos tienen alta disponibilidad para el cultivo de plantas. La FAO, (2) señala hay una disponibilidad baja para la agricultura cuando la CIC es $<10$ meq/100g, disponibilidad media cuando la CIC está entre los parámetros de 10 - 20 meq/100g y un alto nivel de disponibilidad cuando la $\mathrm{CIC}$ es $>20 \mathrm{meq} / 100 \mathrm{~g}$

\section{CONCLUSIONES}

El potencial de Hidrógeno $(\mathrm{pH})$ de la suspensión del suelo y la Conductividad Eléctrica (C. E.) de las muestras están por encima de los parámetros establecidos en la norma nacional, ello dificulta el desarrollo de los cultivos por tratarse de suelos alcalinos.

El Fosforo (P), Potasio (K), Carbono (C), Materia Orgánica y Nitrógeno $(\mathrm{N})$ acumulado en los suelos presentan deficiencias según la clasificación normal de los tipos de terrenos, por lo que tienen que ser tratados para darlo la textura normal y hacerlo apto para uso agrícola.

Elanálisis mecánico delastierrasindica que se tratan de suelos arcillosos, francos arenosos dado que sus valores están fundamentados con los parámetros que establece La FAO.
La Capacidad de Intercambio Catiónico (CIC) demuestra que los valores miliequivalentes por $100 \mathrm{~g}$ (meq/100g) obtenidos en las muestras superan los límites permitidos por La FAO y que se necesita de tratamiento para devolverlo su fertilidad.

La cantidad de Calcio $\left(\llbracket \mathrm{Ca} \rrbracket^{\wedge}(+2)\right)$, magnesio $\left(\left[\mathrm{Mg} \rrbracket^{\wedge}(+2)\right)\right.$, Potasio $\left(\mathrm{K}^{\wedge}+\right)$, concentración de Sodio $\left(\left[\mathrm{Na} \rrbracket^{\wedge}+\right)\right.$ e Hidruro de Calcio ( $\left.\llbracket \mathrm{Ca} \rrbracket^{\wedge}(+2)+\mathrm{H}^{\wedge}+\right)$ concentrado en cada muestra supera el límite permitido por la FAO y para que estén dispuestos para el uso agrícola se necesita de tratamiento.

Los valores de la suma de cationes de los suelos indican que tienen alta disponibilidad para el cultivo de plantas, sin embargo el exceso de componentes químico se debe al uso de las aguas residuales, que han contaminado a los suelos.

\section{REFERENCIAS BIBLIOGRÁFICAS}

1. Ibañez JJ. El suelo y su degradación. Informe del Consejo Superior de Investigaciones Científicas; 2018. From http://www.unescoetxea. org/ext/manual_EDS/pdf/07_suelo_castellano. pdf.

2. FAO. Conservación de suelos y aguas en América Latina y el Caribe. Organización de las Naciones Unidas para la Alimentación y la Agricultura; 2018. From http://www.fao.org/ americas/prioridades/suelo-agua/es/

3. Marjani S, Sagasta JM. Agricultura. ONU; 2018. doi: ISBN 978-92-3-300058-2

4. Noticias Ser.Cajamarca:La otra contaminación Andina; 2015, junio 04. Retrieved Marzo 07, 2021 from http://prevencionar.com.pe/2015/06/04/ cajamarca-la-otra-contaminacion/

5. Guadarrama M, Galván A. Impacto del uso de agua residual en la agricultura. Iberoamericana de Ciencias Biológicas y Agropecuarias; 2015:123. From https://dialnet.unirioja.es/descarga/ articulo/5076403.pdf 
6. Rodríguez N, McLaughlin M, Pennock D. La contaminación del suelo una realidad oculta. Roma: Organización de las Naciones Unidad para la Alimentación y la Agricultura; 2019. From http://www.fao.org/3/I9183ES/i9183es. pdf

7. Mendoza, MA. Evaluación fisicoquímica de la calidad del agua superficial en el Centro Poblado de Sacsamarca, Región Ayacucho. Lima, Perú: Trabajo especial de grado de la Pontificia Universidad Católica del Perú; 2018. From http:// tesis.pucp.edu.pe/repositorio123456789/12256. pdf

8. Larios J, González C, Morales Y. Las aguas residuales y sus consecuencias en el Perú. Saber y Hacer; 2015:09-25. From www.usil.edu.pe/ sites/.../revista-saber-y-hacer-v2n2.2-1-19set16aguas-residuales.pdf

9. Soriano M. Evaluación de la calidad fisicoquímica y microbiológica del agua subterranea utilizada para el consumo humano en el Centro Poblado Pata Pata. Cajmarca. Trabajo especial de grado de la Universidad Privada del Norte; 2018. From http://repositorio. upn.edu.pe/handle/115

10. Díaz $N$, Medina $H$. Contaminación en el suelo y flora por metales pesados en la zona de relaveras de la ex Mina Paredones-San Pablo. Cajamarca. Trabajo especial de grado de la Universidad Privada del Norte;2018. From http:// repositorio.upn.edu.pe/handle/11537/13247

11. Huamaní CF. Determinación del efecto de las aguas servidas sobre el suelo y cultivosen la desembocadura del canal de regadío de las Salinas Bajo. Chancay - Lima. Lima, Perú: Trabajo especial de grado de la Universidad Católica Sedes Sapientiae; 2018. From http://repositorio. ucss.edu.pe/

12. Andrades M, Martínez E. Fertilidad del suelo y parámetros que lo definen. España: Informe de la Universidad de La Rioja. Tercera Edición, 2014. doi:978-84-695-9286-1 